\title{
On the Inscrutability of Logic in Certain Natural Language Contexts
}

\author{
Horst Ruthrof ${ }^{1}$, \\ Philosophy, Murdoch University \\ Perth, Australia
}

\begin{abstract}
The paper opens by defining 'logical universality' as the retention of the propositional content of expressions under any enunciative circumstances. Universality in this sense, the paper claims, cannot be demonstrated in the same manner across different discursive domains and sign systems. Unlike in geometry, arithmetic, algebraic and mathematical logic, where logical universality can be shown to be non-controversial, the concept of universality becomes problematic as soon as natural language terms and syntax are employed. The paper shows the main reasons for this difficulty to lie in the extensional features of natural language, which cannot be adequately captured by intentional means. Intentional descriptions are claimed to apply only to semiotically homogeneous sign systems of a formal kind. Natural language expressions, in contrast, are semiotically heterogeneous, or heterosemiotic, characterised as they are by quasi-perceptual ingredients. Nevertheless, the paper argues, there are three cases in which logical universality can be demonstrated to hold in spite of natural language being employed, one of which is strictly technical language. In contrast, culturally fully saturated natural language use is shown to escape the constraints of logical universality as defined, on the grounds that some of its essential features, such as referential background, reference, and deixis, especially in its implicit form, effectively undermine the retention of identical propositional contents across cultures and time.
\end{abstract}

\section{Introduction}

If logical relations were able to appear outside specific semiotic systems, in pure form as it were, for example as Platonic universals or Kantian noumenal entities, only foolhardy philosophers would be tempted to dispute their universality. Alas, whenever we are dealing

\footnotetext{
${ }^{1}$ H.Ruthrof@murdoch.edu.au
} 
with logic we are engaging at the same time with a specific significatory practice, a sign system, by which logical relations are expressed. The very invention of mathematical logic as an enterprise aiming at univocity and clarity is an acknowledgment of this inevitable semiotic linkage. Under these circumstances, we require a working definition of 'logical universality' suitable to address the two fundamental features of all sign systems: what a sign sequence is about (propositions, reference) and its enunciation (deixis). Let us therefore call a sentence 'logically universal' if its propositional content cannot be altered in its use, that is, by any of its possible deictic applications. The universality of logic then would be characterized and guaranteed by meaning stability under any sort of enunciative circumstances. To be called logically universal, the propositional content of such a sentence would have to remain identical whether we sing it, speak it ironically, utter it in markedly different cultures, under different political circumstances, or recover it from a distant past. In this sense, $x=y->y=x$ for all $x, y$ cannot be regarded as other than logically universal. In Foucauldian terms, we could say that a sentence could be called universal if the statement we make by means of it remains identical no matter under what 'enunciative modalities' it occurs historically or culturally. Foucault, of course, would be horrified at such a prospect. After all, it was precisely his aim to free language from the grip of semantic intentionality and instead locate it squarely in a pragmatics that led him down the winding path at the end of which his readers are finally offered his radically historicised notion of the 'statement'. On the other hand, if Foucault is right, then logic cannot function as universal in any natural language context. Should we be in a position to show that we can generate sentences in natural language whose propositional contents remain unchanged under diverse enunciative circumstances, his pragmatic position will have to be amended. (Foucault 1978) This is why in this paper I want to address the question whether or not and, if so, to what extent, expressions ranging from formal logic to natural language are able to satisfy our opening constraint. Do all these sign systems yield to what Wittgenstein called the 'hardness of the logical must'? (Wittgenstein 2009: §437) As we shall see, answering these questions will require the distinction between at least five distinct cases for which logical universality must be argued in markedly different ways.

\section{Non-controversial logical universality}

When we look at various forms of geometry, algebra, mathematics, formal logic and even strictly technical language, we find that in such artificial sign systems logical universality is something we find difficult to deny. Perhaps surprisingly, this has nothing to do with truth or falsity, or even validity of argument. Whether false or ungrammatical, the claim to logical 
universality appears to hold for the expressions of all formal systems. Why is this so? Let us look at the way formal expressions are construed. The structure of a calculus, for example, typically looks like this. We begin with an arbitrary number of basic signs, a kind of 'alphabet'. Then we design a partial set of terms, made up of signs of the alphabet. This we can call the 'expression' set or the set of 'formulae' of the calculus. Out of these we can construct a partial set which term the 'sentences' of the expression set. And finally there are rules of derivation for every partial set of the formulae of the calculus. Since all of the signs and their combinations in expressions and sentences are uniquely and fully defined, lexical or syntactic murkiness does not occur, the elimination of which was part of the reason for designing the calculus in the first place. In performance, that is, the pragmatics of the calculus, anyone who follows the prescribed procedures will come up with identical results. Enunciation has no effect on the propositional content of any of its formulations which are endlessly transferable without change across time and amongst the most radically different cultures.

This applies to all kinds formal logic, in its algebraic versions from Boole to Frege, its mathematical forms in the contributions by Hilbert, Russell and Whitehead, as well as its further developments to this day, to all algorithms, recursive functions, including such critiques from within the field of logic as by Kurt Gödel and Alonzo Church. Leaving undecidability in certain cases aside, what these varieties of logic have in common is their definitional tightness, which eliminates lexical and syntactic ambiguity, and neutralisation of deixis, which guarantees identity of propositions across pragmatic events. Another reason for their uniformity is that we are dealing here with semiotically homogeneous systems. I will argue later that natural languages, by contrast, are semiotically heterogeneous in virtue of their perceptual and quasi-perceptual traces, which they have inherited from early stages in the evolution of language and which continue to be active ingredients in both lexicon and syntax, a situation that radically separates natural language from the domain of the formal. The remainder of this section addresses two cases in which formal and natural language features are combined.

What happens when a natural language expression is translated into Boolean binary-digital code and sent into the bit stream of our laptop? Are the original sequence of signifiers and its propositional content altered in any way? Just such a case of semantic/pragmatic effects has been argued by Katherine Hayles as a result of what she calls 'flickering signifiers'. (Hayles 
1993) As a matter of fact, try as you may, no such shifts can be demonstrated either at the level of signifiers or at the level of signifieds, except for font changes. (Ruthrof 2000:160161) This should not be surprising. After all, the machinic transformation process which natural language expressions undergo in the computer no more than replicate and economise the input, only to return it in identical shape to the reader. Consequently, we cannot speak of either a change in propositional content or enunciative modification, and so logical universality could be maintained if, that is, it can be successfully argued for all natural language use. A less clear case is the combination of formal signs and natural language expressions as it occurs in the vast arena of specialized industrial communication: technical language. Take for example the note for a welding job, 'Continuous 316 stainless steel welding on both sides of the flange' or the instructions for the use of a tec gun, 'Revs no higher than 1300 for mild steel up to $10 \mathrm{~mm}$ thickness'. What is taking place here when contingency enters the formal, or the formal is imposed on the phenomenal in the guise of natural language terms? If logical universality could not be guaranteed in expressions of technical language technological chaos would be inevitable. Yet whenever chaos does occur in this domain it usually has causes quite different from that of the failure of logical universality. The short answer to the question seems to be that the lexicon ('continuous', 'welding', 'stainless steel') is carefully defined by industrial standards guaranteeing numerical identity and so can be neatly combined with formal features ('316', '1300', '10mm'). No inscrutability here. At the same time, deictic features, or enunciative modalities, are neutralized in the sense that it makes no difference whether we speak such instructions ironically or contemptuously, an aspect of communication that would otherwise seriously affect the propositional content of natural language expressions. Compare the remark ' $\mathrm{A}$ heroic act' uttered by someone who is observing a strong young man bashing an old pensioner. Or consider sentences such as 'I beg your pardon', 'There is only one Jeep', and 'Pardon my French', each of which permits radical changes of propositional contents in diverse pragmatic contexts. 'You bloody bastard' can be an endearing exclamation during an Australian fishing venture or a serious insult, shifts in meaning that have a profound effect on the kind of logical relations we will distil. In contrast, in technical speech, ironic and other reversals of propositional content are denied by its definitional alignment with the formal. As in formal systems, the enunciative function has been neutralised. 


\section{Culture, history and pedagogy}

A quite different situation presents itself in culturally saturated natural language. Here enunciative modalities, as well as referential background, play a vital role. To illustrate some of the complexities we face in such expressions, let me take a recent Chinese example, the new 'guiding principles' or 'mission statement' of the Chinese Education Television in Beijing, as presented to the Chinese government. (Kang 2004) Written in the traditional four characters and four line poetic style, the statement reads in pinyin as follows. Neng li wei xian, Shi shang xui xi, Yi ren wei ben, Fu wu wei he. Literally, this could be rendered in English as 'Ability as priority, Make a fashion of study, People as fundamental, Service as Core.' When asked to elaborate, native Mandarin speakers will respond to these lines with somewhat different readings, such as 'Everybody has to demonstrate their ability, We must catch up with contemporary knowledge, Whatever we do, our focus of attention must always be on people, Serving the people must be our emphasis' or 'Competence is our priority, We must make study fashionable, People are to be regarded as fundamental, Service is our core value.' While what we could call the rough directionality of the message is maintained in these as in a number of similar renderings, we cannot claim to be dealing here with synonymy in any strict sense, let alone a single propositional content that could be transferred across cultures and time. Even in this globally affected and market oriented phrasing of the CETV mission statement, the traditional force of literary language still plays a powerful role. As a result, we are dealing with an indicative grid of terms inviting a range of readings in roughly the same interpretive direction. What is important here is that the Chinese emphasis on interpretive elaboration still contrasts sharply with the techniques of generalisation and formalisation and their goal of tightly controllable propositional contents. This is why when we speak of logical universality in a natural language context differences in cultural history cannot be ignored.

We may wonder why China, in the heyday of its rich tradition, did not grant logic a prominent place in its cultural life. Anyone glancing at summaries of Chinese philosophy is struck by the prominence of Lao-tze, Confucius, Mo-tze, Mencius, the Legalists, and Buddhism, with barely a mention of China's logicians. Though never part of the mainstream of Chinese philosophy, we find documented in the third and second centuries BC a school of logic, the Ming jia, the School of Dialecticians or School of Names. Given the less than encouraging reception of its writers at the time and the outright contempt and hostility to matters of logic since then, it is not surprising that only a tiny portion of their output has survived. Since the dominant strands of Chinese philosophy were concerned mainly with developing rules for 
social conduct in support of the existing hierarchy of political power, obedience and respect for the social ranking order, as well as a meritorious system of education and administrative service, logical argument by itself was regarded throughout the Chinese tradition as impractical, if not frivolous. It apparently never occurred to Chinese thinkers that logic, in conjunction with the advancement of mathematics, could ever prove useful and provide the culture with a special advantage over competing societies. In such a broadly shared frame of mind, the claim that logic was universal would have not have been accepted, except perhaps as universal nonsense.

The two leading figures of the Ming jia movement are Hui Shi (c.380-305 BC) and Gongsun Longzi (320-250 BC). What has remained of Hui Shi's output is a small collection of paradoxes, as well as a record of the high esteem he enjoyed among a select group of contemporaries. His dicta, 'Today I go to Yueh State and arrive there in the past', or 'The myriad things in Nature are both completely similar and completely dissimilar, a state of affairs to be described as a great similarity-in-dissimilarity' well illustrate his emphasis on the relativity of reality as we experience it. Gongsun Longzi wrote mainly about the relation between reality, perception, and language. His observations that 'Fire is not hot' (rather, it is our sensation of heat that makes us think in terms of heat) or 'Eyes do not see' (it is our mind that does so with the assistance of the eye) or his insistence that there is no such thing as a white horse on the grounds that 'whiteness' does not contribute to 'horseness' testify to the kind of original distinctions Gongsun Longzi introduced into Chinese philosophy. His accomplishments in metaphysics apparently included a theory of names and concepts that has been compared to Platonist theorisations of universals. (Wade Baskin 1972:147-148;219224;) But even his practical political engagement and his attempts to show the usefulness of the study of the relation of things and names failed to prevent his achievements from suffering the fate of near oblivion. As Chuangzi, a rival philosopher, is recorded to have remarked about the logicians, 'they were able to subdue other people's mouths, but cannot win their hearts. This is where their narrowness lies'. (Wing-Tsit Chan 1963:233) Does this suggest that logic is simply not applicable to certain cultures? Or can we say that different cultures develop different kinds of logic? And if logic is culture dependent, is logical universality a misguided dream?

Not all natural language use shows up 'propositional inscrutability' as persuasively as does our Chinese 'mission statement'. And in some cases, logical universality can indeed be argued 
in spite of cultural difference. No matter how strange they may at first blush appear, the paradoxes of the 'founder of dialectic', Zeno of Elea, have made it into the first year philosophy classes of most cultures and much with the same results. Why is this so? After all, they emerged from a temporary and culturally distant world and were formulated in a foreign tongue. Can their logic be called universal? And if so, how is that possible? It would seem that if the identity of the propositional content of Zeno's arguments can be retained in all utterance contexts this is so because the double inscrutability of reference and deixis, to broaden Quine's parameters, has been neutralized. (Quine 1964) Indeed, reading Zeno we note that we are able to reduce 'Achilles' and the 'tortoise' to non-living moving entities which we can codify by place holders without loss of meaning. As a result, we can represent the paradox by way of an algorithm. After all, Zeno's extensional entities, such as his chosen names, are no more than a front for intentional, formal principles and so their normal semantic/pragmatic scope is rendered immaterial. Neither the mythological context within which the name Achilles was typically understood by Zeno's Greek audience, nor the real world relations in which tortoises were seen to be embedded, play any role in the logic stakes. Viewed from the perspective of reader as victim, one may feel somewhat manipulated by Zeno's clever use of language. (Habermas 1979) Questions of morality and power aside, we could speak here of a reversal of the procedure of naming as a 'baptism of an object', noted by Wittgenstein (Wittgenstein 2009: §38) and elaborated by Kripke. (Kripke 1980) Certainly, the move via anonymization of natural language terms for logical purposes is the first step in Zeno's reduction of the represented phenomenal world to a non-actual, merely formal spatial matrix, a process that allows him to generate the paradox. From this perspective, Zeno's paradoxes can be viewed as stepping stones in the history of the movement from natural language to formal logic. What is striking in this example, as it is in Kant's Categorical Imperative, is that in spite of its use of what looks like culturally saturated natural language, the generalised content of his language appears to be logically universal.

Taking Zeno as a stand-in for many similar examples, as well as from the perspective of the emergence of geometry and arithmetic, we can say that the path from natural language to the formal is paved with two fundamental reductions: the neutralization of reference and the neutralization of deixis. This process follows two lines of abstraction, two axes that cut across one another; one, the axis ranging from specification to generalisation, the other, the axis with the polarities of materialisation and formalisation. The former permits us to view objects of the world, as well as formal entities, at increasingly or decreasingly levels of specificity or 
generality; the latter permits a process of reduction from 'full' mental-material content to formal emptiness. Generalisation and formalisation (or dematerialisation) are the two basic ways by which we form abstractions. In this reductive trajectory, specific reference in natural language expressions, and the manner in which they are uttered, their enunciative modalities, appear to matter less and less. At a very high level of generalisation and at the point of complete de-materialisation (or formalisation), reference and deixis appear to cease to affect the propositional contents of natural language expressions. The difficult question to answer is where we peg the point at which we can say that a natural language expression has been sufficiently de-referentialised and de-modalised to meet our initial definition in the way it is satisfied by a formal expression such as $(\mathrm{p}->(\mathrm{q}->\mathrm{r}))->((\mathrm{p}->\mathrm{q})->(\mathrm{p}->\mathrm{r})$.

Are we then committed to something like 'contingent universality'?

\section{'Contingent' universality}

Both Leibniz and Kant were aware of the gap in reasoning between the intentional neatness of mathematic logic and the complexities introduced by contingency, even if not precisely in those terms. In order to close the gap, Leibniz built his bridge of zureichender Grund or 'sufficient reason', while Kant went a few steps further by splitting the reasoning process into at least six clearly demarcated procedures, each with its different logical mechanism. (Kant 1956; 1967; 1968) (1) Formal or 'pure' reason is stipulated and definitionally controlled, yielding to verification. (2) Reasoning with empirical concepts introduces fuzziness both at the boundaries of the concepts themselves and the potential infinity of its analysis, a reformulation of 'sufficient reason', yielding to 'falsification'. (Popper 1975). (3) Reasoning in matters of practical ethics borrows stipulated concepts from a quasi-formal system of rules and applies them to the phenomenal world of social action, monitored by community mores. (4) Reflective reason permits judgments of isolated phenomena within complex systems the totality of which escapes us, such that we cannot but stipulate a law not yet given that would govern the instance in question, a bottom-up procedure guided by community expectations. (5) In the absence of such an overall law, Kant proposes a non-Aristotelian telos, or teleological reason, the top-down procedure of an interpretive frame, which we must stipulate if we wish to judge complex phenomena with the help of reflective reason, sanctioned by interpretive communities. (6) Transcendental reasoning, finally, is his second-order logical process of emptying a specific reasoning process of material content, leaving as distilled result the mere bones of the reasoning process itself. (Hintikka 1972) As noted, Kant also added a social component to this immanent scheme, the notion of sensus communis, which 
plays a minimal role in (1), restricted as it is here to a community of logicians and the right to veto, increasing to (4) and (5), where, in the absence of any stability of either subject or predicate, the role of sensus communis as interpretive community becomes all important.

Can logic be called universal in Kant's six forms of reasoning? According to our opening definition of propositional identity only 'pure' reason would qualify. After all, as soon as we involve empirical concepts reflecting phenomenal underdetermination, propositional contents display features of indeterminacy. So, perhaps, logical universality can be rescued only at the level of the formal mechanism underlying his remaining five forms of reasoning. This could go through since those mechanisms do not depend on natural language expressions and can, without loss, be replaced by fully formal sign sequences. However, we are then left with a dilemma: how are we to describe the degree of logical validity of a result of transcendental reasoning such as the Categorical Imperative, reformulated as 'Act in such a way that you would be happy with the rules that informed your action to become general law'? And why is it so difficult for readers of any culture to show that Kant's Enlightenment statement does not apply to non-European societies today or some centuries earlier? Is it because there is a universal logic embedded in it? And if so, what is it that makes it so? Unlike the logical mechanisms of (1) to (5) the Categorical Imperative cannot be translated, without loss, into a merely formal sign sequence. If we replace its natural language terms by mere place holders, removing any reference to their original meaning, we could not reconstruct the thrust of Kant's message. On the other hand, would the retention of its natural language expressions not mean to retain also the contingencies which contradict our initial constraint on logical universality? And yet, short of fully-fledged formalisation, there appears to be a degree of generality operating here that permits Kant to cover all varieties of societies by which we may wish to test the Imperative. What Kant has indeed achieved in the radical generalisation of his transcendental reduction appears to be less a summary of formal ethics than a definition of the social. For the violation of Kant's ethical meta-rule would mean the destruction of any existing society itself. This, I suspect, is why so far none of my students has ever come up with a refutation of the inner logic and practical applicability of that notorious principle, apart from complaining that it does not cater for Schopenhauerian and Nietzschean forms of will and does nothing for their emotions. Any serious denial of the universal validity of the logic of the Imperative would have to invent a 'society' in a possible world in which the bare bones of Kant's Imperative do not apply. 
While Kant succeeded in demonstrating the scope of logical universality via specific reasoning procedures for specific judgments, Frege took a more radical turn. (Frege 1970) By collapsing formal and natural language sense and so stripping 'sense' of the senses, shrinking the ostensive force of Bedeutung as a pointing to scientific reference and eliminating Vorstellung from the lexicon, he was able to reduce sentence meaning to 'pure thought' and the test of two-valued logic. In the ideal of an unambiguously signifying Begriffsschrift (purely conceptual notation) the logical propositional content of natural language expressions is aimed to function just as it does in mathematical logic. The crucial assumption in Frege's scheme is that it is possible in natural language expressions to achieve semantic identity conditions. When we pay close attention to Frege's moves in this enterprise we note that he takes as his starting point the ideality of arithmetic and geometric expressions and then without hesitation, let alone justification, transfers this observations to the domain of natural language expressions, such that the noun phrase 'the evening star' is supposed to function just as do his Euclidian ' $a$ ', ' $b$ ' and ' $c$ '. In other words, Frege makes the massive assumption that the sense of natural language expressions can be equated with formal sense salve veritate. This is what I have referred to elsewhere as 'Frege's error'. (Ruthrof 1993) The question is whether something like the Fregean manoeuvre is required to make the logic of natural languages fit the bill of universality as defined.

It requires a certain kind of conception of language to argue synonymy and truth convention as we find them from Carnap (1967) to Bierwisch (1970) and from Tarski (1956) to Wiggins (1992), respectively. When it comes to synonymy, the assumption for example that active and voice in 'John threw the ball at Jane' and 'The ball was thrown at Jane by John' deliver semantic/pragmatic coextensive identity rests on the assumption that there is no more to natural language than the reflection of a state of affairs. And as long as this extensional side of language can be systematically aligned with its intentional features, as well as equally well controlled by way of definition, synonymy is supposed to pose no problem. What we are denying here, however, is the role of the deictic side of language, its enunciative modalities, an omission that may look insignificant in this example but can play havoc in more complex contexts. The devil here is in the choice of examples. Accounts such as these well suit the description of formal sign systems, but prove less and less satisfactory as the complexity of our examples increases. 
As to truth oriented approaches, one can show that Tarski's 'Convention T' requires framing conditions absent in its original formulation. The attempt by Wiggins to revive the Fregean truth criterion rests on the stipulation of a 'literal' meaning which very much looks like an intentional entity. (Wiggins 1992: 66) Donald Davidson's application of a reformulated 'Convention T' for truth-conditional semantics shares this problem and in addition hides the fact that propositional checking mechanisms are second-order operations and as such parasitic on resemblance relations and so on meaning. (Ruthrof 2013) If we follow Quine here, a path I recommend, such stipulations cannot get off the ground. (Quine 1964) As far as natural language is concerned, we should instead settle for what Quince calls 'domestic meanings'. (Quine 1993: 53ff.) This has serious consequences for the claim to logical universality in natural language. Assumptions as to the intentional nature of meaning in natural languages surface in the description of a number of specific features. Recursivity is foregrounded by Chomsky, much as if it operated more or less the same as in formal signification. (Chomsky 1957) Predicability in natural language has likewise been constructed as a formal system. (Keil 1981). Much the same can be said about grammaticality and syntax. The fundamental problem in all such descriptions is that they are a little bit like formal tools employed to measure the coastline of Australia, never to yield quite the same result. The more complex our examples, the less satisfying the outcome. Unless we play rough shot with our initial constraint of logical universality, 'is logic universal?' does not look likely to be answerable in the affirmative, at least not in general.

\section{Natural language and intentional entities}

When Quine rejected the view that natural language could in any way be adequately described in terms of intension, he revived a theme that we find towards the end of Kant's first Critique where empirical concepts are sharply distinguished from their mathematical cousins. Kant here makes two important observations. One concerns conceptual boundaries, that is, the problem of the firmness of the definitional delimitations that stabilize the scope of the concepts of natural language. As far as empirical concepts are concerned, Kant says, their boundaries are 'never secure'. (KrV 725ff.) The second seminal observation in this section can be regarded as a consequence of the first in that it curtails the degree to which our conceptual analysis is able in principle to successfully pursue the task of stabilization. Here Kant emphasizes the sobering point that any such analysis 'will never be complete'. If Kant is right on these two points, since natural language relies heavily on empirical concepts, the intentional description of natural language is misguided in whatever form. For if the 
boundaries of empirical concepts are wobbly and our analysis of the nature of such concepts cannot be halted logically but only by the practical decision of a Leibnizian sufficient reason, then natural language expressions fundamentally violate the definitional neatness of any notion of intension. Since the sense of a term viewed in its intentional context would depend only on its definition, without taking into consideration its extensional scope, its semantic/pragmatic range of application, natural language terms violate the very principle on which the distinction of intension and extension is based. We cannot define a natural language expression while disregarding the classes of all objects covered by it, and so its 'intension' is always already contaminated, as it were, by its 'extensions'. In using a phrase such as 'social responsibility' we are never quite in the position of a logician who first defines ' $x$ ' as ' $2 y$ ' and then provides his intentional entities with an extension such as ' $y=$ the width of my thumb'. Furthermore, while the formal expressions have logical universality, the semantic/pragmatic scope of 'social responsibility' varies from culture to culture, as do its explicit and implicit enunciative modalities. In the case of culturally saturated language expressions we find ourselves from the outset in a holistic frame of meaning negotiation within social limits, involving reference, referential background, as well as explicit and implicit deixis.

Quine famously elaborates the implications of the failure of intentional description in theories of meaning, among other things, in terms of inadequate notions of meaning, a critique of synonymy, and the 'inscrutability of reference'. (Quine 1964) Yet referential inscrutability is by no means an all or nothing affair. Rather we should speak of degrees of inscrutability depending on two factors, one, what kind of sign system we are talking about and, two, to what kind of use a sign system is put. I briefly comment on each of these. (1) Reference functions somewhat differently according to different semiotic systems. In formal signification with formal reference, Quine's inscrutability is absent since we are dealing with the intentional entities of two systematically aligned axiomatically and definitionally governed structures. Nor can reference in a technical blueprint justifiably called inscrutable where intentional contexts constrain reference such that interpretation beyond acts of numerical identification is strictly curtailed. By contrast, in culturally fully saturated signification, such as natural language, Quine's critique bites, with devastating consequences for a range of positions in the philosophy of language. (2) The specific use to which a sign system is put in pragmatic contexts is another factor that plays a role in deciding on the degree to which referential opacity makes reference fixing problematic. (Ruthrof 1992:4-5) This applies most forcefully to natural language. And since there is no such thing as a non- 
pragmatic context, including attempts at aligning natural language with formal semantics, we cannot without falsification investigate language outside such contexts. Given this caution, whenever natural language is employed in its full range of cultural involvements, rather than as a technical, reduced version, inscrutability of reference poses a serious problem for the philosophy of language well beyond Quine's intervention. For Quine's analysis is vulnerable to the charge that had he embraced a broader frame of analysis, including that of nonverbal semiosis in which all natural languages are crucially embedded, he would have been able to offer solutions to his 'gavagai' problematic and related observations in a more satisfactory manner. (Ruthrof 2003) Nevertheless, we should embrace his rejection of intensions as appropriate tools for the description of natural language and his emphasis on our difficulties in securing reference.

Referential inscrutability in Quine's critique, however, remains limited in a few crucial respects. I suggest that the view of natural language held by Quine, as by the majority of analytical language theorists is too limited. For the broadly shared assumption that the semantic and pragmatic sides of natural language are well described by linguistic expression, sense, and reference, with some slack given to deictic notions in speech act theory and presuppositions in Gricean analysis, is simply too narrow. What is missing is first the fact that natural language is not only used in acts of referring in Strawson's sense and so has reference as a result of use, (Strawson 1950; Evans 1982) but that its lexicon also has what I have called referential background, that is the typical way linguistic expressions indicate portions of a cultural world prior to pinpointing specific references. Frege's 'morning star' is a prime example. Without the shared referential background of typical mornings indicated in the phrase we would not be able to argue the link to the reference 'Venus'. Likewise, the 'evening star'. Second, the traditional analysis of marked deixis needs to be supplemented by an investigation of implicit deixis, the typical way in which a culture utters its terms. When 'xin $b u$ ' in traditional Chinese culture was typically translated as 'Ministry of Justice', it is useful to be reminded that the more literal translations of 'pain inflicting ministry' or 'department of physical punishment' much better reflect the manner in which the vocabulary of law, legal processes, and legislation were spoken and thought about. Given this history and in spite of a massive recent campaign of introducing law oriented thinking and processes into contemporary Chinese consciousness, matters of law are still being viewed suspiciously and with an eye to the traditional conception of the law as punitive. Without such larger notions of referential background and implicit deixis our analysis misses two vital components. 
If referential and deictic complications of this sort are absent in formal languages yet forcefully present in natural languages, what accounts for the difference? I drew attention earlier to the difference between semiotic homogeneity and semiotic heterogeneity, the former characteristic of artificial languages, the latter being typical of their natural language counterparts. Formal sign systems are semiotically homogeneous in the sense that they strictly employ terms from within one and the same domain, formal sense, 'pure thought', or intentionality. In contrast, natural languages are fundamentally tied to their extensional contexts via referential background, specific reference, as well as marked and implicit deixis. At the same time, and this is a crucial point to note, this extensionality is semiotically heterogeneous, characterized as it is by the necessary activation of nonverbal sign systems, such as olfactory, gustatory, haptic, tactile, visual and other sign clusters in the performance of language. This necessary linkage in the meaning making process in natural language between expressions and their extensional nonverbal components is as important as it is undertheorized. An approach which attempts to account for the extensional side of natural language in terms of nonverbal semiosis and quasi-perceptual traces can be found in Ruthrof (1997; 2000; 2007; 2009).

Because of the different forms of signification that are active in meaning events in natural language, the semiotic homogeneity of formal signs does not apply; without it, natural language expressions escape strictly definitional constraints. This is why one misses the point if one treats natural language extensionality by intentional means. In order to realize full cultural reference and deixis, or even their sufficient equivalents, the user of a natural language typically activates its verbal signifiers by means of nonverbal Vorstellung, the collective of mental states appropriate for each speech situation. It is well to remember at this point that Vorstellung, as mental modification of perception, is by definition multisemiotic and therefore heterosemiotic and cannot, without falsification, be translated into something formal, as for instance 'propositional attitudes'. So, if natural language cannot be described satisfactorily in terms of intentional entities and contexts and if their reference and deixis are complicated in the manner indicated, the claim to logical universality would seem to have suffered a severe setback. 


\section{Conclusion}

In light of what has been said, we face five distinct kinds of semiotic contexts for which the claim of logical universality must be argued differently. Two of them strike me as uncontroversial because it can be shown that their propositional contents do not change under diverse enunciative conditions and so satisfy our opening definition. (1) One is formal logic, such as mathematical and symbolic logic; the other (2) technical language proper, that is, language in which deixis is fully neutralised and reference strictly delimited. The other three cases share the medium of natural language but are problematic in that they must be carefully distinguished not only from (1) and (2), but also amongst themselves. All three share some logical relations as part of their structure and meaning while those relations are veiled to different degrees by the characteristics of natural language. (3) In the third case we need to read 'through' their natural language components to distil the logical relations hidden behind the cultural ingredients of reference, referential background and deictic features. This is done by generalising and formalising those natural language features to the degree to which they were neutralised in the original design of the logical relations to be expressed. Logical universality is disguised as natural language narrative. What we have to do as readers to see through the manipulation is to transform the extensional character of the natural language text into its intentional skeleton. Here, then we have a situation of natural language use where an intentional approach is indeed not just relevant but the appropriate path to argue logical universality. Such is the case with Zeno's paradoxes, as we have seen. (4) There are cases in which sentences in natural language present a logical sequence while retaining a degree of referential and deictic mental material, yet nevertheless qualify in terms of our opening definition. They do so as a consequence of the high level of semantic generality at which they are formulated. However, it must remain a moot point whether it is possible to design a rule by which we could determine precisely at what level of abstraction such a qualification is justified. Suffice it to suggest that this level must be meta-cultural, an abstraction from the specifics of any kind of culture, to be able to produce a rule of subsumption. Referential and deictic reduction in this type of language use then is achieved not by pretence but rather by a process of abstraction laid bare for the reader, as in the transcendental reduction of Kant's Categorical Imperative. Natural language use according to (3) and (4) however is by no means self-evident, but requires decisions based on interpretation. Yet the involvement of interpretive acts complicates the matter. For the interpreter would have to know in advance and in principle what precisely satisfies the criteria of referential and deictic neutralisation, as well as what degree of generalisation of natural language phrases suffices to meet the 
universality requirement. An alternative route is the one taken in this paper, that is, to ask whether a natural language statement (in the non-Foucauldian sense) retains its propositional content under any thinkable enunciative circumstances. If it does, it can claim logical universality; if it does not, it cannot. If it can, then its referential and deictic reductions are deemed sufficient; if it doesn't they are not. The Leibnizian move here may rescue the procedure from vicious circularity, but still leaves our interpretive problematic unsolved, a task to be taken on elsewhere. (5) No such manoeuvres, however, are likely to shore up the claim to universality of logical relations embedded in fully fledged natural language use. For it follows from what has been said that logical universality cannot be secured in any instance of natural language use where reference, referential background, marked deixis and implicit or cultural deixis remain specific, that is, are neither sufficiently neutralised nor lifted to a level of sufficient generality that would eliminate cultural, historical and other specifications. Such is the case of a vast bulk of natural language expressions. No matter how charitably we apply our opening constraint of continued identity of propositional contents under diverse utterance conditions, in natural languages employed in their full cultural settings, no such condition can be consistently argued. Neither does such natural language use yield to intentional description, nor can their extensional relations to the nonverbal complexities of cultures rescue them from inscrutability, an arch enemy of logical universality. So we are forced to conclude that while mathematical and other formal kinds of logic, $(1,2)$ including such interventions as those made by Gödel and Church (Gödel 1962; Church 1958), satisfy our initial definition and while logical relations can be regarded charitably as universal in the kind of natural language use described in (3) and (4), any such compliance must remain a logician's dream for all cases of (5). As we have seen, this is not the result of a failure of the ideality of logical relations, the universality of which has not be doubted, but rather of the inscrutability of logic in heterosemiotic, culturally fully saturated natural language contexts.

\section{References}

Baskin, Wade (1972) Classics in Chinese Philosophy. New York: Philosophical Library.

Bierwisch, Manfred (1970) 'Semantics' in New Horizons in Linguistics, ed. John Lyons. (Harmondsworth: Peguin), pp.166-184.

Carnap, Rudolph (1968) Meaning and Necessity. Chicago: University of Chicago Press. 
Chomsky, Noam (1957) Syntactic Structures. The Hague: Mouton.

Chu, Yingchi (2007) Chinese Documentaries: From Dogma and Polyphony. London: Routledge.

Church, Alonzo (1958) Introduction to Mathematical Logic. Princeton: Princeton Universisty Press.

Evans, Gareth (1982) The Varieties of Reference. Oxford. Clarendon.

Foucault, Michel (1978) The Archaeology of Knowledge. London: Tavistock.

Frege, Gottlob (1970) 'On Sense and Reference' in Translations from the Philosophical Writings of Gottlob Frege, ed. Peter Geach and Max Black. (Oxford: Basil Blackwell), pp.56-78.

Gödel, Kurt (1962) On Formally Undecidable Propositions of Principia Mathematic and Related Systems I. London: Oliver and Boyd.

Habermas, Jürgen (1979) 'Universal Pragmatics' in Communication and the Evolution of Society (London: Heinemann), pp.1-68.

Hayles, Katherine (1993) 'Virtual Bodies and Flickering Signifiers' October, 66, 69-91.

Hintikka, Jaakko (1972) ‘Transcendental Arguments: Genuine and Spurious’ Nous 6, 274-81.

Kang, Ning (2004) CETV Program Reform. Beijing: CETV.

Kant, Immanuel (1956) Kritik der reinen Vernunft. Hamburg: Felix Meiner.

Kant, Immanuel (1967) Kritik der praktischen Vernunft. Hamburg: Felix Meiner.

Kant, Immanuel (1968) Kritik der Urteilskraft. Hamburg: Felix Meiner.

Keil, Frank (1981) 'Constraints on Knowledge and Cognitive Development' Psychological Review 88, 197-227.

Kripke, Saul (1980) Meaning and Necessity. Cambridge, Mass.: Harvard University Press.

Popper, Karl Raimund (1975) The Logic of Scientific Discovery. London: Hutchinson.

Quine, Willard van Ormond (1964) Word and Object. New York: MIT Press.

Quine, Willard van Ormond (1993) Pursuit of Truth. Cambridge, MA: Harvard University Press. 
Ruthrof, Horst (1993) 'Frege's Error’ Philosophy Today 37, 306-17.

Ruthrof, Horst (1997) Semantics and the Body: Meaning from Frege to the Postmodern.

Toronto: University of Toronto Press.

Ruthrof, Horst (2000) The Body in Language. London: Cassell.

Ruthrof, Horst (2005) 'The Semiotics of Intercultural Exchange' Semiotica 157, 1/4,

387-410.

Ruthrof, Horst (2007) 'Principles of Corporeal Pragmatics' The Public Journal of Semiotics, 1/2, 12-30. http://www.semiotics.ca/issues/pjios-1-2.pdf.

Ruthrof, Horst (2009) 'Justifications for a Perceptually Oriented Theory of Language' Signs - International Journal of Semiotics, 3,1-29. ISSN: 1902-8822. http://vip.db.dk/signs/Articles.htm

Ruthrof, Horst (2013) 'Metasemantics and Imaginability' Language Sciences (in press).

Strawson, P.F. (1950) ‘On Referring’ Mind 59, 320-44.

Tarski, Alfred (1956) 'The Concept of Truth in Formal Languages' in Logic, Semantics, Metamathematics. (Oxford: Clarendon), pp. 152-227.

Wiggins, David (1992) 'Meaning, Truth-Conditions, Propositions: Frege’s Doctrine of Sense Retrieved, Resumed and Redeployed in Light of Certain Recent Criticisms', Dialectica 46, 61-90.

Wittgenstein, L. (2009) Philosophical Investigations, trans. Anscombe, G.E.M., Hacker, P.M.S. and J. Schulte. Basil Blackwell, Oxford. 